\title{
Chronological Observations of Iris Flocculi in a Japanese Family with Thoracic Aortic Aneurysm and Dissections
}

\author{
Yuka Mori Noriyasu Hashida Kazunobu Asao Kazuichi Maruyama \\ Kohji Nishida \\ Department of Ophthalmology, Osaka University Graduate School of Medicine, \\ Osaka, Japan
}

\section{Keywords}

Iris flocculi $\cdot$ ACTA2 $\cdot$ Familial thoracic aortic aneurysm and dissections

\begin{abstract}
Iris flocculi, a type of hereditary iris pigment epithelial cyst, have been reported in association with smooth muscle $\alpha-2$ actin (ACTA2) gene as the causative gene of familial thoracic aortic aneurysm and dissections (FTAAD). The purpose of the report was to examine morphological changes in the shape of flocculi and iris features over time by comparing infants to adults with FTAAD combined with iris flocculi. A Japanese family with FTAAD and bilateral iris flocculi and the Arg149Cys gene mutation was included. A slit-lamp photograph and anterior segment optical coherence tomography (AS-OCT) were used to evaluate the structure and location of iris flocculi. AS-OCT was also used to measure the internal shape and iris thickness of the dilated pupil muscle. A morphological change in the cyst was confirmed to be existent in the youngest cases. Pigment discoloration and iris atrophy of the iris body were observed in all cases. Besides, a decrease in iris thickness was observed with AS-OCT measurement. Changes across generations in iris flocculi occurred in a Japanese family with TAAD. ACTA2 gene abnormalities may cause iris atrophy and decrease thickness in addition to iris flocculi in early life. The prognosis is poor when FTAAD is combined with iris flocculi, and prevention of cardiovascular disease is necessary based on earlier findings of its emergence.
\end{abstract}


Background

Iris flocculi are primary iris pigment cysts characterized by congenital multiple pigmented epithelial cysts located at the pupillary border in association with a thoracic aortic aneurysm and dissections (TAAD) [1]. Guo et al. [2] reported that a mutation in smooth muscle $\alpha$-actin (ACTA2) was a risk haplotype and reported that an allelic variant of ACTA2, Arg149Cys, caused familial TAAD (FTAAD), iris flocculi, and livedo reticularis with autosomal dominant inheritance. Pathologically, iris flocculi consist of pigment epithelium and goblet cell components that arise from iris pigment cell layers [3]. Another investigator reported that previous ocular manifestations heralded cardiovascular disorders, such as TAAD [4]. Therefore, it is very important to detect ocular findings during childhood and prevent the development of the cardiovascular disease. We report one familial case of iris flocculi and TAAD caused by a mutation in ACTA2. We investigated the chronological changes that occurred in iris flocculi and monitored structural changes with anterior segment optical coherence tomography (AS-OCT) through the third consecutive generation.

\section{Case Presentation}

Eight eyes of four patients among Japanese familial cases of bilateral iris flocculi and TAAD were included in this study. The structures and locations of iris flocculi were evaluated with a slit-lamp photograph and AS-OCT. The thickness of the iris at the dilator muscle regions was measured with AS-OCT (CASIA ${ }^{\circledR}$, TOMEY Co. Ltd., Japan) according to a previous report [5]. Briefly, the thickness was measured at a middle position of the iris between the scleral spur and the pupillary margin in an AS-OCT image with the provided software.

\section{Case 1}

A 56-year-old woman who was the mother of Cases 2 and 3 was found to have a heterozygous mutation (Arg149Cys) in ACTA2. This case was one of the cases previously reported [3]. Bilateral iris flocculi were recognized at the pupillary margin (Fig. 1a, e). Some of these were found to be collapsed. Iris atrophy and depigmentation were also observed (Fig. 2a, e). Fundus examinations showed no abnormalities. Surgery could not be performed because she died of aortic dissection at the age of 56 years in 2008.

Case 2

A 50-year-old man who was the older brother of Case 3 had hypertension and had been operated on for aortic dissection twice at the ages of 25 and 27 years. A heterozygous mutation (Arg149Cys) in ACTA2 was identified. Iris abnormality had been noted while he was at primary school. Visual acuity was $20 / 20$ in both eyes, and intraocular pressure was normal. A slit-lamp examination showed bilateral iris flocculi (Fig. 1b, f) and iris atrophy/depigmentation (Fig. 2b, f). Photos were taken when he was 38 years old. Fundus examinations showed no abnormalities. AS-OCT showed cystic components that lined the pupillary margin of the iris (Fig. 3a, b). The thickness of the iris at the dilator muscle regions was measured on an ASOCT image and found to be $385 \mu \mathrm{m}$ at the right and $319 \mu \mathrm{m}$ at the left iris. Surgery was performed to remove the iris flocculi, and the postoperative course was uneventful. 


\section{Case Reports in Ophthalmology}

Case 3

A 44-year-old woman presented who had complained of disturbances in bright spaces since childhood. This case was also one of the cases described previously [3]. She had no previous ophthalmic history but had been operated on for TAAD at the age of 28 years. A heterozygous mutation (Arg149Cys) in ACTA2 was identified. At the first medical examination, visual acuity was $20 / 20$ in both eyes, and intraocular pressure was normal. Photos were taken when she was 32 years old. A slit-lamp examination showed bilateral iris flocculi at the pupillary margin and iris atrophy at the dilator muscle region (Fig. 1c, g, Fig. 2c, g). The thickness of the iris at the dilator muscle regions was measured on an AS-OCT image and found to be $320 \mu \mathrm{m}$ at the right and $310 \mu \mathrm{m}$ at the left iris. Fundus examinations showed no abnormalities. FTAAD was diagnosed based on the existence of iris flocculi, a familial history, and the mutation in ACTA2. Surgery was performed because cystic formation obstructed the patient's vision bilaterally in brightly illuminated surroundings. Ocular surgery was finally performed and resulted in symptomatic improvement.

Case 4

A 12-year-old male patient who was the eldest son of Case 1 was found to have a heterozygous mutation (Arg149Cys) in ACTA2. A slit-lamp examination showed iris atrophy and some tubers in the iris that were assumed to represent an earlier stage of iris flocculi (Fig. 1d, h, Fig. 2d, h). The structures of the iris were examined with AS-OCT, which showed budding flocculi (Fig. 3c, d). His iris thickness was $316 \mu \mathrm{m}$ at the right and $271 \mu \mathrm{m}$ at the left iris. Evaluation of cardiovascular disease was performed, but no abnormalities were observed. His younger brother did not have a mutation in ACTA2 or any iris abnormality or cysts.

\section{Discussion}

We examined chronological changes in iris flocculi associated with a mutation (Arg149Cys) in ACTA2 by comparing clinical images obtained in young and adult cases. We found that the morphological changes that occurred in the cysts showed they emerged in childhood and later developed the complete structure of the flocculi. In addition, it is likely that iris atrophies might occur in addition to iris flocculi. ACTA2 has been reported to be a causative gene of FTAAD $[2,4]$. It has been reported that there are fewer smooth muscle cells and elastic fibers in affected patients, in whom proteoglycans are increased due to the degeneration of blood vessels in FTAAD [2]. Since the $\alpha$-actin protein is expressed in the pupil sphincter and pupil dilator muscle [5], structural changes might occur in both lesions due to genetic abnormalities. As the expression of ACTA2 has been found to initiate from the embryonic stage, even in a young case, the primordia of flocculi might be noticeable at the pupillary margin.

We observed that there were changes in the pupil dilated muscle, and all of the present cases with iris flocculi showed discoloration of the pigment of the iris, indicating suspected iris atrophy. It is likely that during growth and aging, the loss of function of the ACTA2 protein resulted in its accumulation, consequential iris flocculi around the pupil sphincter region, and iris atrophy. Considering the morphological changes observed in the iris, previous investigators reported that in 2,231 eyes of normal subjects, the thickness of the pupil dilator muscle was $446.9 \pm 92.6 \mu \mathrm{m}$ [5]. Because the numbers of cases were small, statistical significance could not be evaluated; however, iris thickness was found to be decreased in our case series. Chamney et al. [4] also reported that among patients with familial aortic dissection, cases re- 
quiring surgery for vascular lesions were more common than were observed for those without iris flocculi. Thus, in patients with a mutation in ACTA2, it is possible that the reduction in pupil dilator muscle leads to a reduction in iris thickness. When iris flocculi are observed, it is important to suspect a mutation in ACTA2, to carry out a whole-body examination, and to take a familial history to predict/prevent cardiovascular disease.

In conclusion, we report a case of FTAAD with iris flocculi. A mutation in ACTA2 might cause iris atrophy in addition to iris flocculi. Iris atrophy might emerge precedential to iris flocculi, periodical ophthalmic examinations are necessary.

\section{Statement of Ethics}

Written informed consent was obtained from the patient for publication of this case report and any accompanying images. A copy of the written consent form is available for review by the Editor of this journal. The Institutional Review Board of the Osaka University Medical School approved the research protocol, and the procedures conformed to the tenets of the Declaration of Helsinki.

\section{Disclosure Statements}

The authors declare that there are no conflicts of interest regarding the publication of this paper.

\section{Funding Sources}

The authors did not receive funding.

\section{Author Contributions}

Y.M. participated in drafting the manuscript and collection, analysis, and interpretation of the data. N.H. participated in the diagnosis and treatment of the patient and drafting the manuscript and revised the manuscript. K.A. participated in drafting the manuscript and collection, analysis, and interpretation of the data. K.M. participated in drafting the manuscript and collection, analysis, and interpretation of the data. K.N. critically reviewed the manuscript and reviewed the literature. All authors consent to their names being published in this report and insist on the intellectual honesty and validity of data provided. All authors read and approved the manuscript.

\section{References}

1 Lewis RA, Merin LM. Iris flocculi and familial aortic dissection. Arch Ophthalmol. 1995 Oct;113(10):1330-1.

2 Guo DC, Pannu H, Tran-Fadulu V, Papke CL, Yu RK, Avidan N, et al. Mutations in smooth muscle alpha-actin (ACTA2) lead to thoracic aortic aneurysms and dissections. Nat Genet. 2007 Dec;39(12):1488-93.

3 Hashida N, Ohguro N, Morimoto Y, Oiki E, Morisaki H, Morisaki T, et al. Ultrastructural appearance of iris flocculi associated with a thoracic aortic aneurysm and dissections. Br J Ophthalmol. 2009 Oct;93(10):140910. 


\section{Case Reports in Ophthalmology}

\begin{tabular}{l|l}
\hline Case Rep Ophthalmol 2019;10:397-402 \\
\hline DOI: 10.1159/000504432 & $\begin{array}{l}\text { @ 2019 The Author(s). Published by S. Karger AG, Basel } \\
\text { www.karger.com/cop }\end{array}$ \\
\hline
\end{tabular}

Mori et al.: Chronological Observations of Iris Flocculi in a Japanese Family with Thoracic Aortic Aneurysm and Dissections

4 Chamney S, McGimpsey S, McConnell V, Willoughby CE. Iris Flocculi as an ocular marker of ACTA2 mutation in familial thoracic aortic aneurysms and dissections. Ophthalmic Genet. 2015 Mar;36(1):86-8.

5 Prata TS, Palmiero PM, Angelilli A, Sbeity Z, De Moraes CG, Liebmann JM, et al. Iris morphologic changes related to alpha(1)-adrenergic receptor antagonists implications for intraoperative floppy iris syndrome. Ophthalmology. 2009 May;116(5):877-81.
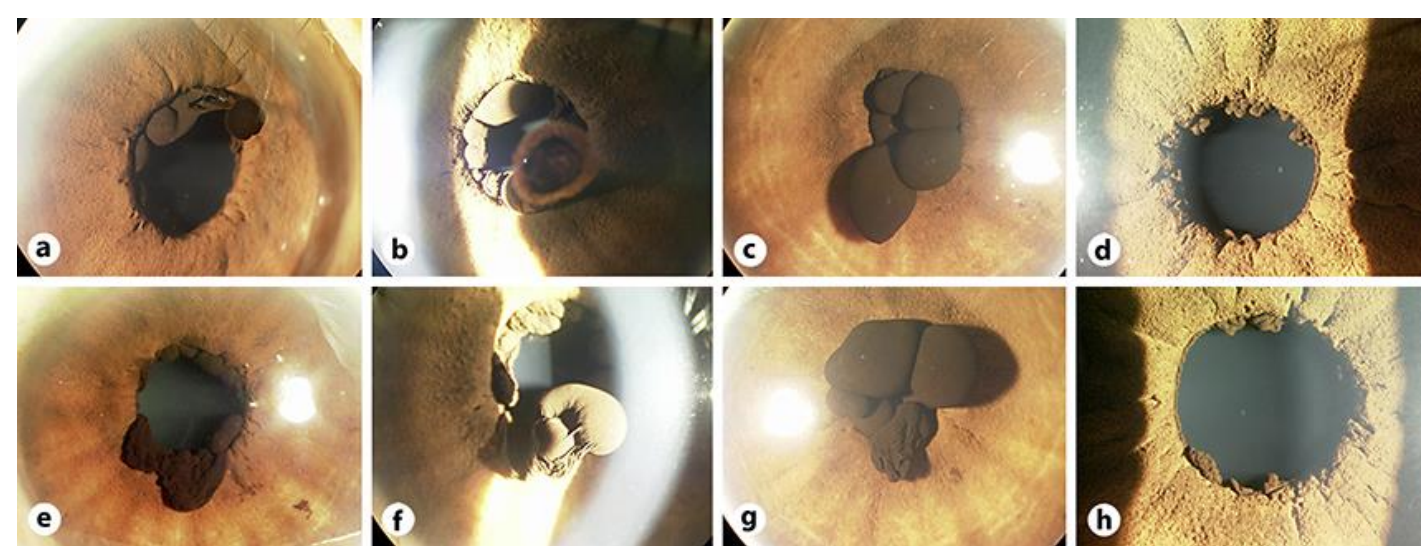

Fig. 1. Slit-lamp photographs of Case 1 (a, e), Case 2 (b, f), Case $3(\mathbf{c}, \mathbf{g})$, and Case 4 (d, h). The pupillary margin is lined with pigmented iris flocculi $(\mathbf{a}-\mathbf{c}, \mathbf{e}-\mathbf{g})$, some of them showing partly collapsed with shrinking (a, e), with anterior synechiae (b) and budding $(\mathbf{d}, \mathbf{h})$.

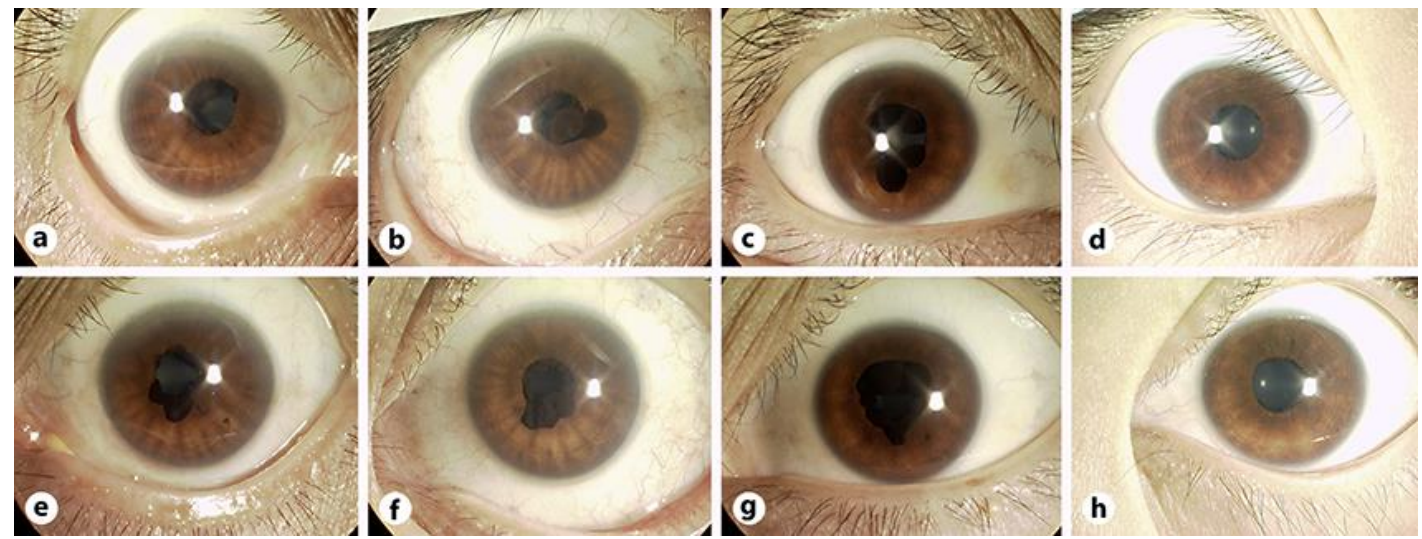

Fig. 2. Slit-lamp photographs of Case 1 (a, e), Case 2 (b, f), Case $3(\mathbf{c}, \mathbf{g})$, and Case $4(\mathbf{d}, \mathbf{h})$. Iris atrophy was observed in all cases. Morphological changes such as iris depigmentation and budding of flocculi emerged from a time of childhood $(\mathbf{d}, \mathbf{h})$. 


\section{Case Reports in Ophthalmology}

\begin{tabular}{l|l}
\hline Case Rep Ophthalmol 2019;10:397-402 \\
\hline DOI: 10.1159/000504432 & $\begin{array}{l}\text { ○ 2019 The Author(s). Published by S. Karger AG, Basel } \\
\text { www.karger.com/cop }\end{array}$ \\
\hline
\end{tabular}

Mori et al:: Chronological Observations of Iris Flocculi in a Japanese Family with Thoracic Aortic Aneurysm and Dissections

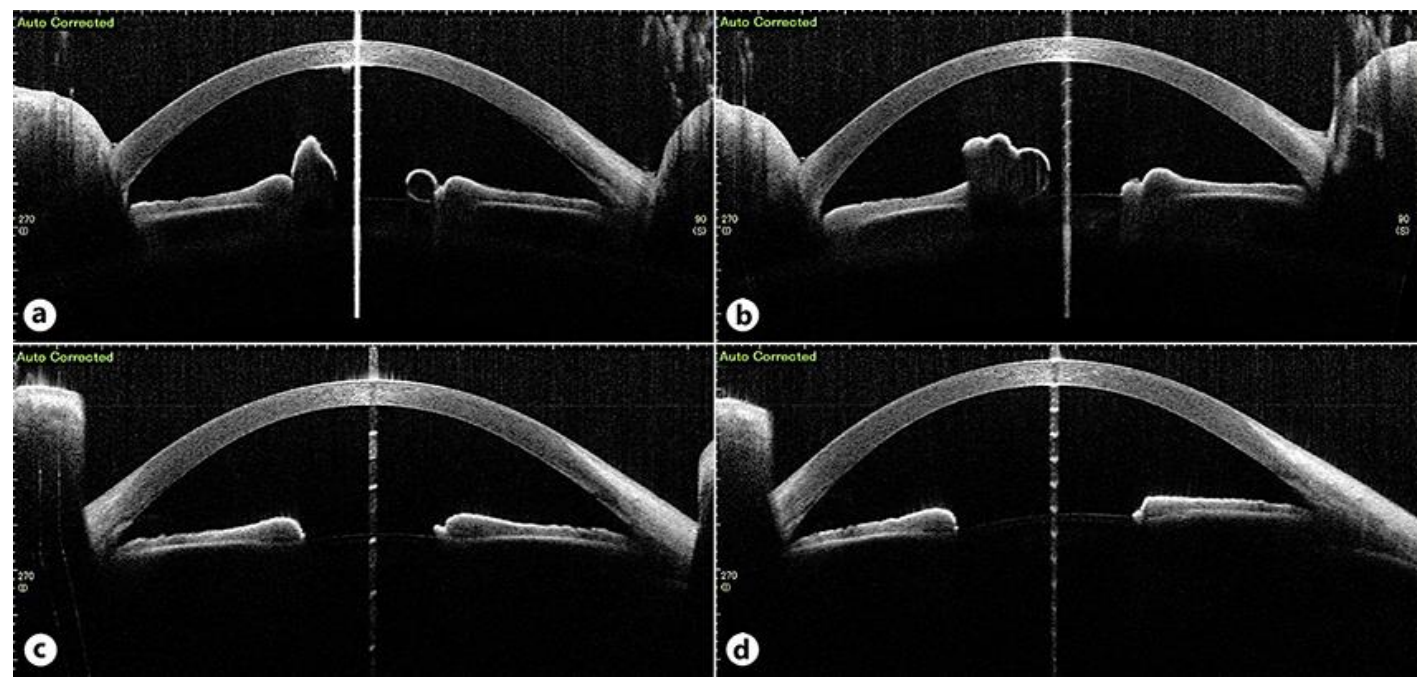

Fig. 3. Representative images of AS-OCT showed cystic components lining the pupillary margin of the iris in Case $2(\mathbf{a}, \mathbf{b})$ and budding of flocculi in Case $4(\mathbf{c}, \mathbf{d})$. A decrease of thickness was observed with measurement. 